\title{
First observation of multiple volume reflection by different planes in one bent silicon crystal for high-energy protons
}

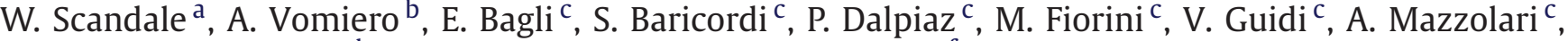 \\ D. Vincenzi ${ }^{c}$, R. Milan ${ }^{d}$, Gianantonio Della Mea ${ }^{e}$, E. Vallazza ${ }^{f}$, A.G. Afonin ${ }^{g}$, Yu.A. Chesnokov ${ }^{g}$,
} V.A. Maisheev ${ }^{g}$, I.A. Yazyning ${ }^{g}$, V.M. Golovatyuk ${ }^{h}$, A.D. Kovalenko ${ }^{h}$, A.M. Taratin ${ }^{\text {h, }}{ }^{*}$, A.S. Denisov ${ }^{\mathrm{i}}$, Yu.A. Gavrikov ${ }^{i}$, Yu.M. Ivanov ${ }^{i}$, L.P. Lapina ${ }^{i}$, L.G. Malyarenko ${ }^{i}$, V.V. Skorobogatov ${ }^{i}$, V.M. Suvorov ${ }^{i}$,

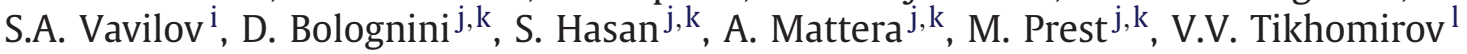

${ }^{a}$ CERN, European Organization for Nuclear Research, CH-1211 Geneva 23, Switzerland

${ }^{\mathrm{b}}$ INFM-CNR, Via Vallotti 9, 25133 Brescia, Italy

c INFN Sezione di Ferrara, Dipartimento di Fisica, Università di Ferrara, Via Saragat 1, 44100 Ferrara, Italy

d INFN Laboratori Nazionali di Legnaro, Viale Università 2, 35020 Legnaro (PD), Italy

e Dipartimento di Ingegneria dei Materiali e Tecnologie Industriali, Università di Trento, Via Mesiano 77, 38050 Trento, Italy

${ }^{\mathrm{f}}$ INFN Sezione di Trieste, Via Valerio 2, 34127 Trieste, Italy

${ }^{g}$ Institute of High Energy Physics, Moscow Region, RU-142284 Protvino, Russia

h Joint Institute for Nuclear Research, Joliot-Curie 6, 141980, Dubna, Moscow Region, Russia

i Petersburg Nuclear Physics Institute, 188300 Gatchina, Leningrad Region, Russia

j Università dell'Insubria, Via Valleggio 11, 22100 Como, Italy

k INFN Sezione di Milano Bicocca, Piazza della Scienza 3, 20126 Milano, Italy

${ }^{1}$ Research Institute for Nuclear Problems, Belarusian State University, 220030 Minsk, Belarus

\section{A R T I C L E I N F O}

\section{Article history:}

Received 21 July 2009

Received in revised form 7 October 2009

Accepted 11 November 2009

Available online 14 November 2009

Editor: M. Doser

PACS:

61.85.+p

\section{A B S T R A C T}

Multiple volume reflection by different planes in a bent silicon crystal with its $\langle 111\rangle$ axis orientation close to the beam direction was observed for the first time for $400 \mathrm{GeV} / c$ protons at the CERN SPS. The proton beam was deflected to the side opposite to the crystal bend by an angle of about $67 \mu$ rad, which is five times larger than in a single volume reflection by the (110) bent planes. The registered efficiency of one side deflection was about $84 \%$. It was shown that multiple volume reflection transforms to a single volume reflection when the orientation angle of the $\langle 111\rangle$ axis relative to the beam direction is increased.

(C) 2009 Elsevier B.V. All rights reserved.
Volume reflection (VR) of ultra-relativistic charged particles by bent crystal planes predicted in [1] was observed recently for protons with different energies [2-4]. The VR dependence on the crystal bend radius was investigated in [5]. Recently volume reflection was also observed for negative particles, $150 \mathrm{GeV} / c \pi^{-}$mesons, in our experiment (will be published).

Volume reflection with a high efficiency up to $98 \%$ occurs in a wide interval of the crystal orientation angles determined by the crystal bend angle [4]. This property is an important advantage of volume reflection versus channeling. However, the maximum value of the VR deflection angle $\theta_{\mathrm{vr}}$ is only about $1.4 \theta_{c}$, where $\theta_{c}=\left(2 U_{0} / p v\right)^{1 / 2}$ is the critical channeling angle, $p, v$ are the particle momentum and velocity and $U_{0}$ the well depth of the crystal potential averaged along the planes. The beam deflection angle can

\footnotetext{
* Corresponding author.

E-mail address: Alexander.Taratin@cern.ch (A.M. Taratin).
}

be increased in the set of subsequent volume reflections using a few bent crystals - multiple volume reflections (MVR). The deflection efficiency of such crystal system can be high. It was more than $90 \%$ for the deflection of $400 \mathrm{GeV} / c$ protons due to MVR in five crystals [6].

Only volume reflection of particles by bent crystallographic planes normal to the crystal bend plane was considered in [2-6]. However, VR of particles by the skew planes having acute angles relative to the bend plane was also registered in our experiments with $400 \mathrm{GeV} / c$ protons. It was predicted in [7] that particles entering a bent crystal with a small angle with respect to a crystal axis can be deflected due to the set of volume reflections by different planes crossing the axis. This effect of multiple volume reflections in one crystal (MVR OC) should increase the deflection angles of particles by several times.

This Letter presents the observation of multiple volume reflection of $400 \mathrm{GeV} / c$ protons in a bent silicon crystal when its $\langle 111\rangle$ axis is oriented at a small angle with the beam. The deflection of 


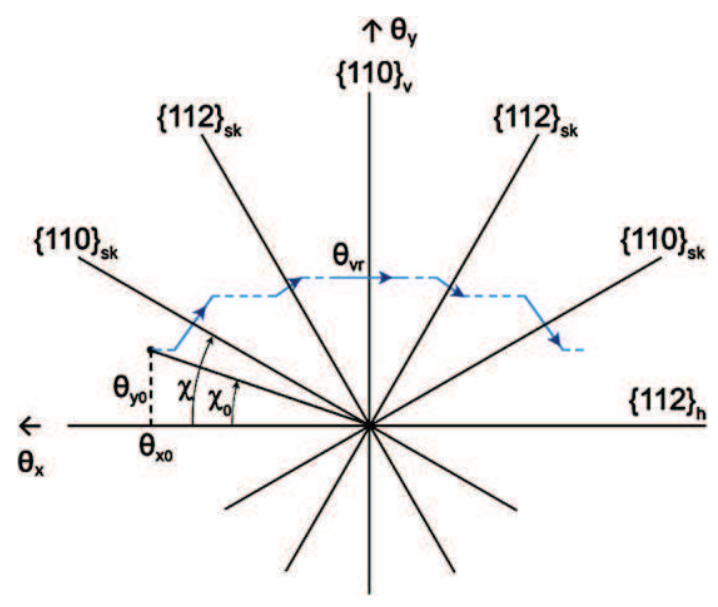

Fig. 1. (Color online.) Angular space around the $\langle 111\rangle$ axis of a silicon crystal. Only main crystal planes are shown. Simplified picture of a proton angle evolution due to MVR in the crystal bent along the vertical $(110)_{v}$ plane is also shown (by blue) in the commoving coordinate system. Volume reflections of the particle occur in the tangency areas with the planes and its momentum direction changes stepwise by the angle $\theta_{\mathrm{vr}}$ in the direction normal to the crystal planes.

protons with the efficiency larger $80 \%$ by the angle in five times larger than the deflection angle in a single volume reflection has been observed.

The possibility of subsequent reflections of particles by bent planes inside one crystal can be understood by considering the angular space near the bent crystal axis. Fig. 1 shows the main planes, (110) and (112), passing through the $\langle 111\rangle$ axis of a silicon crystal. It shows also in a simplified way in the commoving system the angle evolution of the particle performing MVR. In the commoving coordinate system, which rotates with the particle velocity along the arc of the radius $R$ around the crystal curvature center, the crystal plane orientation is unchanged. For the case of a bent crystal Fig. 1 shows the angular space at the crystal entrance. It should be shifted by the bend angle $\alpha$ to the left for the crystal exit.

The crystal used in the experiment was bent along the vertical $(110)_{v}$ plane, that is the crystal bend plane is coincident with the horizontal (112) $h$ plane. The crystal bend angle $\alpha$ determines the angular interval for changing the particle momentum direction relative to the crystal planes during the particle passage through the crystal. The angular distances between the crystal planes reduce quickly when we approach to the axis, in our case along the vertical $(110)_{v}$ plane. Therefore, the angular interval $\alpha$ determined by the crystal bend can cover more and more crystal planes crossed the axis. So, when a particle crosses the crystal its momentum can be subsequently tangential to the crystal planes with different angles $\chi$ to the crystal bend plane.

In the tangency area with all considered planes volume reflection of the particle occurs. As a result the particle momentum direction changes stepwise by the angle $\theta_{\mathrm{vr}}$ in the direction normal to the plane (see Fig. 1). So, the particle reflection from a skew crystal plane with the inclination angle $\chi$ gives it both the horizontal projection of the deflection $\theta_{x}=\theta_{\mathrm{vr}} \sin \chi$ and the vertical one $\theta_{y}=\theta_{\mathrm{vr}} \cos \chi$. The horizontal projections of the VR deflections from all crystal planes are directed to the side opposite to the crystal bend. They are summed and the overall deflection is increased several times. In contrast the vertical projections of the VR deflections from the skew planes symmetrically disposed around the vertical plane have opposite signs and cancel each other. So, when the particle trajectory is symmetric in the angular space of the commoving system it does not receive any vertical angular deflection.
It should be noted that the bend radii of the skew planes are larger than the radius $R$ for the $(110)_{v}$ plane, $R_{\text {sk }}(\chi)=R / \sin \chi$. For the $(110)_{\text {sk }}$ planes $R_{\text {sk }}\left(30^{\circ}\right)=2 R$. The VR deflection angle $\theta_{\mathrm{vr}}$ is increased with increasing the crystal bend radius according to [5]. Therefore, for the $(110)_{\text {sk }}$ planes it should be larger than for the $(110)_{v}$ plane. However, for the considered bend radius $R=$ $11.43 \mathrm{~m}$ the increase is not large, $\Delta \theta_{\mathrm{vr}} \approx 5 \%$. The (112) planes are more weak than the (110) planes. The VR deflection angle by the (112) planes should be $\theta_{\mathrm{vr}}(112) \approx 0.75 \theta_{\mathrm{vr}}(110)$.

The particle momentum direction at the crystal entrance shown by the point $\left(\theta_{x o}, \theta_{y_{0}}\right)$ in Fig. 1 should satisfy some conditions, which are optimal to observe the MVR effect [7]. The tangency point of the incident particle momentum with the vertical $(110)_{v}$ bent plane should be in the middle of the crystal, that is

$\theta_{x o}^{*}=0.5 \alpha$,

to realize the contributions from the skew planes both on the right and on the left from the vertical crystal plane. At a given angle $\theta_{x o}$ the vertical angle of the particle momentum $\theta_{y o}$ determines the number of the skew planes participating in the MVR. Volume reflection occurs from all planes with the inclination angles $\chi$ to the crystal bend plane

$\chi>\chi_{o}=\operatorname{arctg}\left(\theta_{y_{0}} / \theta_{x_{0}}\right)$,

when the condition (1) is satisfied. So, the condition

$\theta_{y o}^{*}=0.5 \theta_{x o}^{*}$ with $\chi_{o}=26.6^{\circ}$

realizes the contributions of the main $(110)_{s k}$ skew planes, for which $\chi=30^{\circ}$ and $150^{\circ}$, and all others with $\chi_{0}<\chi<180^{\circ}-\chi_{0}$.

The experimental setup was the same described in [5]. Four microstrip silicon detectors, two upstream and two downstream of the crystal, were used to detect the particle trajectories with an angular resolution of $3 \mu \mathrm{rad}$, which is limited by the multiple scattering of particles in the detectors and the air.

A $70 \times 4 \times 0.5 \mathrm{~mm}^{3}$ silicon strip with the largest faces parallel to the (110) planes and with the side faces parallel to the (111) planes was fabricated according to the technologies [8,9] and placed in a vertical position. The beam entered the crystal through its side face. The preliminary alignment using a laser beam allowed making the largest faces to be parallel to the beam direction with accuracy better than 0.5 mrad. The anticlastic curvature produced along the crystal width $T=4 \mathrm{~mm}$ due to its mechanical bending along the length was used for the beam deflection in the horizontal plane (see Fig. 1 in [5]).

The measured RMS deviation values for the horizontal and vertical divergence of the beam were $\sigma_{x}=(11 \pm 0.06) \mu \mathrm{rad}$ and $\sigma_{y}=(9.13 \pm 0.03) \mu \mathrm{rad}$, respectively. A high precision goniometer allowed orienting the crystal in the horizontal and vertical planes with the accuracy of $2 \mu \mathrm{rad}$. The scan of the horizontal orientation angles of the crystal $\theta_{h}$ was first performed. It allowed finding the angular position of the vertical (110) planes $\theta_{h}^{p}$, which is characterized by the maximal deflection efficiency of protons in channeling regime. The deflection angle of channeled protons was measured to be about $350 \mu \mathrm{rad}$. The deflection angle is determined by the crystal bend angle $\alpha$, therefore $\alpha=350 \mu \mathrm{rad}$. This gives for the average value of the anticlastic bend radius $R=T / \alpha=11.43 \mathrm{~m}$. Then the scan of the vertical orientation angles of the crystal $\theta_{v}$ allowed finding the angular position of the $\langle 111\rangle$ axis $\theta_{v}^{a}$.

For the MVR observation the crystal orientation was selected to realize the incident beam direction relative to the $\langle 111\rangle$ crystal axis with $\theta_{x o}^{*}=0.5 \alpha \approx 170 \mu \mathrm{rad}$ and $\theta_{y o}^{*}=0.5 \theta_{x o}^{*}=85 \mu \mathrm{rad}$ according to (1), (3). Fig. 2a shows the intensity distribution of the proton beam passed through the crystal in the horizontal and vertical deflection angles. Let us note that the deflection due to 

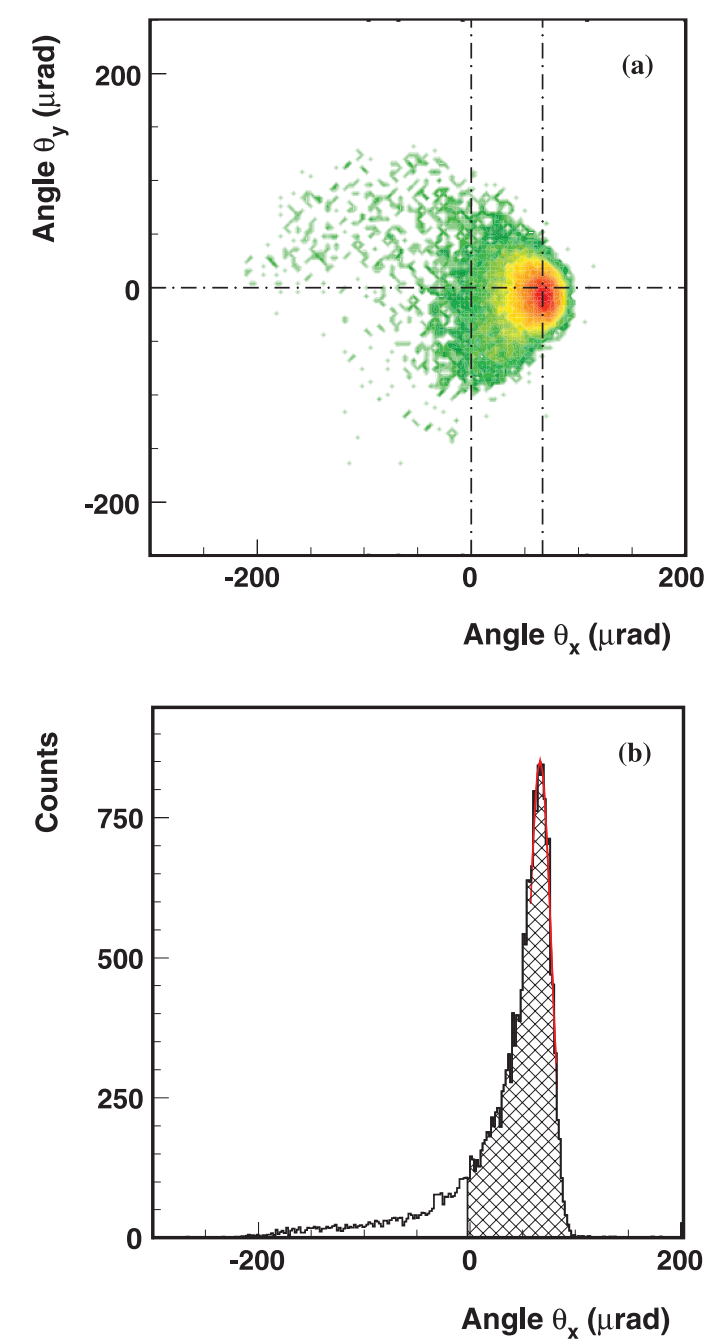

Fig. 2. (Color online.) (a) Intensity distribution of the proton beam in the horizontal and vertical deflection angles acquired due to multiple volume reflections from different planes in one bent silicon crystal around the $\langle 111\rangle$ axis. Maximum position is shown by dot-dashed line. (b) The horizontal projection of the distribution. The distribution part with $\theta_{x}>0$ (about 84\%) is hatched. Gaussian fit gives for the maximum position $\theta_{\mathrm{mvr}} \approx 66.5 \mu \mathrm{rad}$.

volume reflections was written as a positive one for the experimental data in contrast with Fig. 1. The horizontal projection of the distribution is shown in Fig. 2b. Gaussian fit gives the value $\theta_{m}=$ $(66.53 \pm 0.27) \mu \mathrm{rad}$ for the maximum position. This angle is about five times larger than the VR deflection angle of protons by the vertical $(110)_{v}$ plane, whose value $\theta_{\mathrm{vr}}=(13.35 \pm 0.17) \mu \mathrm{rad}$ was registered for the beam incidence with the angle $\theta_{y_{0}}^{\mathrm{vr}} \approx 1.3 \mathrm{mrad}$. So, the additional deflection of protons due to volume reflections by the skew planes is a value of about $4 \theta_{\mathrm{vr}}$. The $(110)_{\mathrm{sk}}$ and $(112)_{\text {sk }}$ planes shown in Fig. 1 give approximately a half of this value and the other skew planes, which are more weak and not shown, give a second half. The deflection efficiency to the side opposite to the crystal bend is $P_{d}\left(\theta_{x}>0\right)=(83.86 \pm 0.26) \%$. The distribution tail to the bend side is caused by the volume capture of protons into the channeling regime when they pass the tangency areas with different crystal planes. A small shift of the maximum position in the vertical direction (see Fig. 2a) can be explained by an incomplete compensation of the contributions from the symmetric skew planes.

Two scans of the crystal orientation angles along the horizontal and vertical directions, which change the incident beam direction

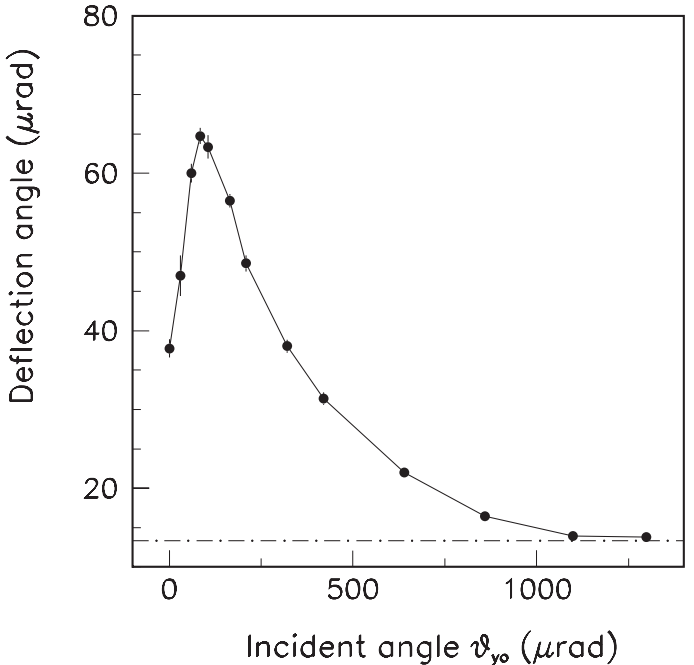

Fig. 3. Dependence of the MVR deflection angle $\theta_{\text {mvr }}$ on the vertical angle $\theta_{y_{0}}$ of the incident beam relative to the $\langle 111\rangle$ axis. Dot-dashed line shows the deflection angle for a single VR from the $(110)_{v}$ plane, $\theta_{\mathrm{vr}} \approx 13.35 \mu \mathrm{rad}$.

near the point $\left(\theta_{x 0}^{*}, \theta_{y o}^{*}\right)$, showed that the selected orientation is really close to the optimum one. Fig. 3 shows the dependence of the MVR deflection angle value $\theta_{\mathrm{mvr}}$, the distribution maximum position, on the vertical angle of the incident beam $\theta_{y o}$ relative to the axis direction. The angle $\theta_{y_{0}}$ changes the number of the skew planes, at which VR of protons occurs. The deflection angle has a maximal value at the angle $\theta_{y_{0}}=\theta_{y_{0}}^{*}=85 \mu \mathrm{rad}$. For $\theta_{y_{0}}<\theta_{y_{0}}^{*}$, reducing the angles of protons with the $\langle 111\rangle$ atomic strings forming the planes destroys the stability condition of the particle trajectories along the planes. This reduces the VR deflection angles. So, the optimum conditions for MVR (1), (3) should be added by the stability condition for particle trajectories along the crystal planes

$\theta_{y_{0}} \gg \psi_{1}, \quad \psi_{1}=\sqrt{\frac{4 Z_{1} Z_{2} e^{2}}{p v d}}$,

where $\psi_{1}$ is the critical angle for axial channeling along the $\langle 111\rangle$ axis, $Z_{1}$ and $Z_{2}$ are the atomic numbers of the incident particle and the crystal atom, $d$ is the interatomic spacing in the string. For $400 \mathrm{GeV} / c$ protons $\psi_{1}=20.7 \mu \mathrm{rad}$. So, for the considered crystal orientation the condition ( 4 ) is satisfied, $\theta_{y_{0}}^{*} \approx 4 \psi_{1}$. With increasing $\theta_{y_{0}}$ in the range $\theta_{y_{0}}>\theta_{y_{0}}^{*}$ the angular interval of the crystal bend $\alpha$ includes less and less number of the skew planes. So, at a sufficiently large orientation angle with the axis, for our case at $\theta_{y_{0}}>1 \mathrm{mrad}$, we approach to the case of a single VR from the vertical plane when the deflection angle of protons $\theta_{d}=\theta_{\mathrm{vr}}$. The observed dependence can be described in the planar potential approximation for the angles $\theta_{y_{0}} \geqslant \theta_{y_{0}}^{*}$ and in the multi-axes potential approximation for all values of $\theta_{y_{0}}[7,10]$.

Fig. 4 shows the MVR deflection angle distribution of protons for a parallel incident beam with the crystal parameters and its orientation as in the experiment, which was obtained by simulation (1). The model of atomic string lattice [10] with the atomic potential and electron density obtained in the Doyle-Turner approximation for the atomic scattering factors was used for simulation. The distribution looks like the experimental one (Fig. 2b). Gaussian fit gives the value $\theta_{m}=(69.77 \pm 0.1) \mu \mathrm{rad}$ for the distribution maximum. The calculated efficiency of the deflection to the side opposite to the bend $P_{d}\left(\theta_{x}>0\right)=(86.65 \pm 0.11) \%$. For comparison, the calculated distribution of deflection angles for the single VR by the vertical $(110)_{v}$ plane is also presented here (2); its maximum value $\theta_{\mathrm{vr}}=(14.06 \pm 0.03) \mu \mathrm{rad}$. 


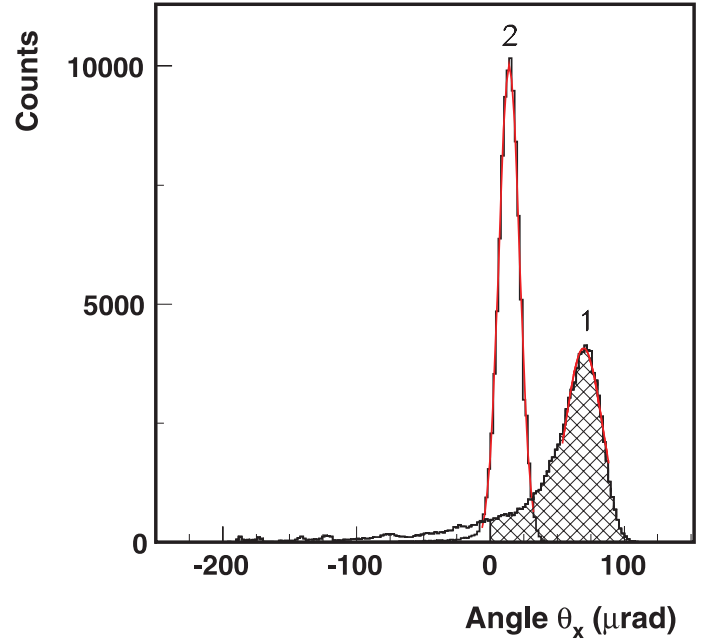

Fig. 4. (Color online.) MVR deflection angle distribution of protons (1) received by simulation. Histogram 2 shows the distribution for a single reflection by the $(110)_{v}$ plane.

Our experiment gives the observation of the effect of multiple volume reflections from different crystallographic planes inside one bent silicon crystal for high-energy protons. The additional contribution of particle reflections from the skew planes allows increasing the deflection angle about five times. The efficiency of one side deflection is higher than $80 \%$.

The MVR process in one bent crystal enriches possibility of crystal deflectors for steering the beams of high-energy charged particles, although it requires using a two axis goniometer. The
MVR deflection angle should be proportional to the atomic number of the crystal $Z_{2}$ and $p^{-1 / 2}$. So, instead of using some multi-crystal system with subsequent reflections of particles [6] one bent $\mathrm{Ge}$ crystal $\left(Z_{2}=32\right)$ can be used to obtain the MVR deflection angle larger than $100 \mu \mathrm{rad}$ for $400 \mathrm{GeV} / c$ protons, which is already sufficient for the purposes of the accelerator beam collimation and extraction.

\section{Acknowledgements}

We are grateful to Professor L. Lanceri (INFN \& University of Trieste) who provided the tracking detectors, to V. Carassiti and M. Melchiorri for the design and fabrication of the crystal holders. We acknowledge partial support by the INFN NTA-HCCC and MIUR 2006028442 projects, the INTAS program, the Russian Foundation for Basic Research Grants 05-02-17622 and 06-02-16912, the RF President Foundation Grant SS-3057-2006-2, the "Fundamental Physics Program of Russian Academy of Sciences" and the Grant RFBR-CERN 08-02-91020.

\section{References}

[1] A.M. Taratin, S.A. Vorobiev, Nucl. Instrum. Methods Phys. Res., Sect. B 26 (1987) 512.

2] Yu.M. Ivanov, et al., Phys. Rev. Lett. 97 (2006) 144801

[3] Yu.M. Ivanov, et al., JETP Lett. 84 (2006) 372.

[4] W. Scandale, et al., Phys. Rev. Lett. 98 (2007) 154801.

[5] W. Scandale, et al., Phys. Rev. Lett. 101 (2008) 234801.

[6] W. Scandale, et al., Phys. Rev. Lett. 102 (2009) 084801.

[7] V. Tikhomirov, Phys. Lett. B 655 (2007) 217.

[8] S. Baricordi, et al., Appl. Phys. Lett. 91 (2007) 061908.

[9] S. Baricordi, et al. J. Phys. D: Appl. Phys. 41 (2008) 245501.

[10] A.M. Taratin, S.A. Vorobiev, Phys. Lett. A 115 (1986) 401 